(polypeptide chains) can be seen. A number of exciting applications of two-dimensional electrophoresis to various biomedical problems have been published during the last 2-3 years (e.g. refs. 16,17).

In our laboratory we now use capillary GC-MS computer methods for determination of low molecular weight compounds and two-dimensional electrophoresis for multiprotein analyses. Figure 2 shows an example of such a multicomponent analysis where a small piece (approximately $10 \mathrm{mg}$ ) of a brain tumor has been separated into over 1100 constituents of low and high molecular weight. With the invention of twodimensional electrophoresis and when combining this technique with capillary GC-MS, one is now approaching what might be called 'total analyses'. It is not difficult to predict that this type of multicomponent analysis is likely to have considerable impact on medical research and diagnosis in the future.

\section{References}

1 Stanbury, J. B., Wyngaarden, J. B. and Fredrickson, D. S. (eds) (1978) in The Metabolic Basis of Inheriled Disease, McGraw-Hill, New York, 4th edn

2 Jellum, E. (1977) J. Chromatogr. 143, 427

3 Sjøvall, J. and Axelson, M. (1979) J. Steroid Biochem. 11, 129

4 Zlatkis, A. and Kim, K. (1976) J. Chromatogr. 126, 475

5 Spiteller, M. and Spiteller, G. (1979) J. Chromatogr. 164, 253

6 Heller, S. R., McCormick, A. and Sargent, T. (1980) in Waller, G. R. and Dermer, O. C. (eds) Biochemical Applications of Mass Spectrometry, first supplementary volume, Wiley-Interscience, New York

7 Gates, S. C., Smisko, M. J., Ashendel, G. L., Young, N. D., Holland, J. F. and Sweeley, C. C. (1978) Anal. Chem. 50, 433

8 Horning, E. C. and Horning, M. G. (1971) J. Chromatogr. Sci. 9,129
9 Halpern, B. (1981) in Biomedical Applications of Gas Chromatography-Mass Spectrometry, CRC critical reviews, Chemical Rubber Publishing Company, Cleveland, U.S.A. (in press)

10 Waller, G. R. and Dermer, O. C. (eds) (1980) in Biochemical Applications of Mass Spectrometry, first supplementary volume, Wiley-Interscience, New York

11 Mitruka, B. M. (1975) in Gas Chromatographic Applications in Microbiology and Medicine, John Wiley \& Sons, New York

12 Wold, S. and Sjøstrøm, M. (1977) in Kowalski, B. (ed.), Chemometrics: Theory and Applications, ACS Symposium series, no. 52, American Chemical Society, Washington, D.C. p. 243

13 Jellum, E. and Wold, S. to be published.

14 Jellum, E., Stokke, O. and Eldjarn, L. (1973) Anal. Chem. 45, 1099

15 O'Farrel, P. H. (1975) J. Biol. Chem. 250, 4007

16 Anderson, N. G. and Anderson, N. L. (1979) Behring Inst. Mitt. 63, 169

17 Anderson, N. G., Anderson, N. L. and Tollaksen, S. L. (1979) Clin. Chem. 25, 1199

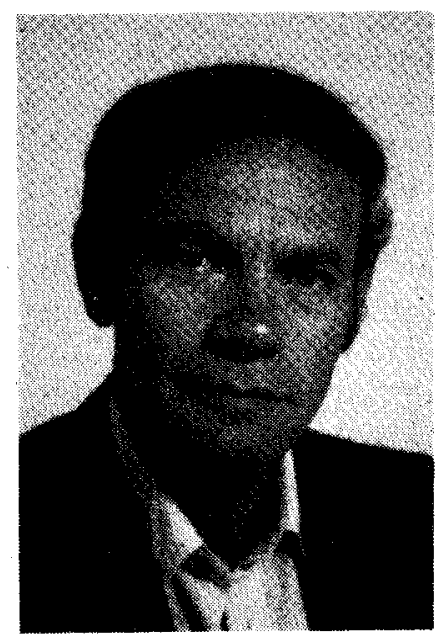

Egil Jellum received his Ph.D. from the University of Oslo in 1966 and is currently Chairman of the Institute of Clinical Biochemistry at the university hospital Rikshospitalet, Oslo, where he has been since 1963 . In 1969 he spent 6 months as a visiting scientist at Stanford University.

\title{
EXAFS, determination of short range order and local structures in materials
}

\author{
D. C. Koningsberger and R. Prins \\ Laboratory for Inorganic Chemistry and Catalysis, Eindhoven University of Technology, Eindhoven, The Netherlands
}

\begin{abstract}
Extended X-ray Absorption Fine Structure (EXAFS) is a powerful method of determining short range order and local structures in materials using $X$-ray photons produced by a synchrotron light source, or in-house by a high intensity rotating anode $X$-ray generator. The technique has provided valuable structural information on disordered solids (such as metal-on-support catalysts), amorphous solids, semiconductors and the environment of metals in metallo-enzymes.
\end{abstract}

\section{The structure of materials}

An important property of matter is its structure and it was indeed a landmark in science when Von Laue and Bragg discovered that the long range structure of materials could be measured with the aid of X-ray diffraction. However, the X-ray diffraction technique can only be used for materials which have a structural order over some distance. The structure of materials which have a lack of such a long range order and show 0 165-9936/81/0000-0000/\$02.50 a structural order over a short range only, such as amorphous materials, cannot be solved by X-ray diffraction. Furthermore, local structures arcund ions in solutions and defects in lattices escape detection.

Recently, a new technique has emerged which is capable of filling up the gap in the need for short range order structure determinations. This technique is called EXAFS (Extended X-ray Absorption Fine Structure). In principle, it furnishes information on the 
number and types of neighbouring atoms, interatomic distances and mean square deviations of neighbouring atom positions about the equilibrium values. In addition, such information can independently be derived for all the constituent atoms or ions in a material. It will be clear that the EXAFS technique is especially useful for the determination of short range order in non-crystalline materials like glasses, catalysts, solutions and of the local structure around atoms or ions in solids or solutions. In this article examples of such applications of the EXAFS method will be given, together with a short description of the technique and its underlying principles.

\section{EXAFS modulation of the X-ray absorption process}

In the EXAFS technique the X-ray absorption spectrum of a material is measured over an extended energy range in the neighbourhood of the absorption edge of one particular element of the material involved. The principles of the measurement of the X-ray absorption coefficient are given in Fig. 1. The modulation of the X-ray absorption coefficient observed on the high energy side of the absorption edge constitutes the Extended X-ray Absorption Fine Structure (EXAFS). The fine structure starts at an energy of about $30 \mathrm{eV}$ beyond the edge and extends over a range of about $1000 \mathrm{eV}$. This kind of fine structure is observed in the absorption coefficient $(\mu)$ of molecules and condensed matter (see Fig. 2). Gaseous atoms show an absorption coefficient $\mu_{0}$ without these modulations. The fractional modulation $\chi(k)$ is defined as:

$$
\chi(k)=\frac{\mu(k)-\mu_{0}(k)}{\mu_{0}(k)}
$$

with

$$
k=\left[2 m\left(\hbar \omega-E_{0}\right) / \hbar^{2}\right]^{1 / 2}
$$

( $k$ : wave vector, $\hbar \omega$ : energy of incident photon, $E_{0}$ : threshold energy, $m$ : mass of electron and $\hbar=(h / 2 \pi)$ : constant of Dirac).

The physical process which causes these oscillations of the absorption coefficient originates in the ejection of an electron from an atom (e.g. a 1 s core electron) by

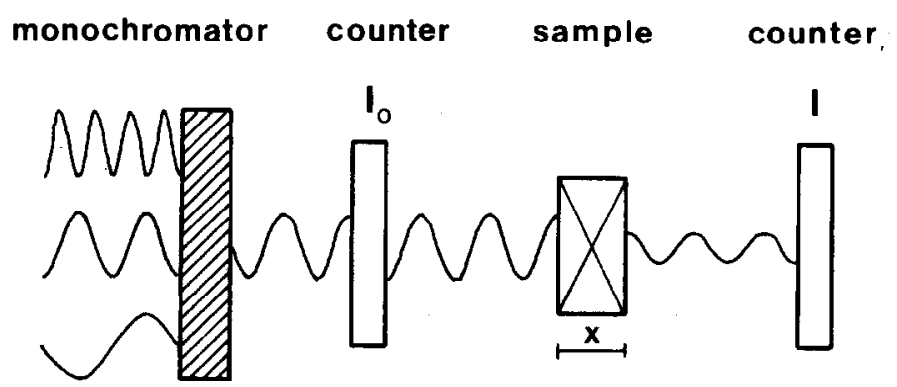

$$
\text { Absorption }=u x=\ln \left[\frac{I_{0}}{l}\right]
$$

Fig. 1. Schematic representation of the measurement of the absorption coefficient.

- Circle no. 3 on advertising enquiry form

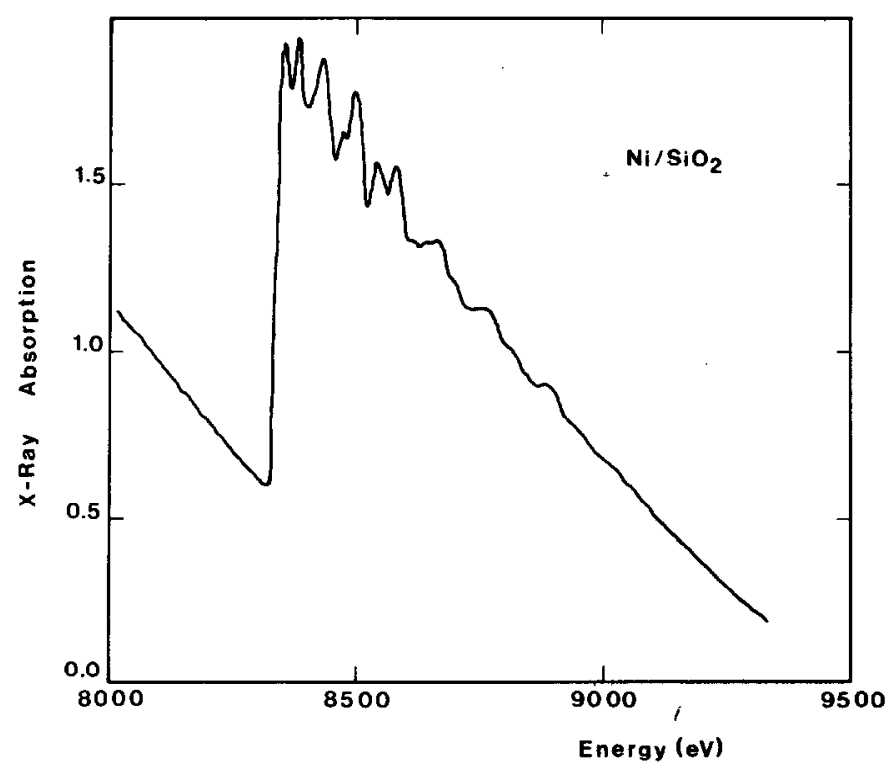

Fig. 2. X-ray absorption spectrum of a nickel metal catalyst finely dispersed $\left(\mathrm{d} \sim 40 \mathrm{~A}\right.$ ) on $\mathrm{SiO}_{2}$. (Metal loading 5 tet \%). Measuring temperature $125 \mathrm{~K}$.

an impinging $\mathrm{X}$-ray photon. In this way a bound electron can be excited to a continuum state when the energy $E=\hbar \omega$ of the incident photon is higher than $E_{0}$, the binding energy of the core electron (Fig. 3). The resulting photoelectron can be represented by an outgoing spherical wave with wavelength $\lambda=(2 \pi / k)$, which is determined by the photon energy $\hbar \omega$ and the core state energy $E_{0}$ (formula 2 ). This wave will be scattered back by the neighbouring atoms and subsequently by the now ionized atom, a process resulting in an interference wave pattern. By varying the energy $E$ of the incident photons, the wavelength of the ejected photoelectron changes, which can cause constructive or destructive interference. The interference wave pattern determines the final state wave function of the outgoing electron and hence the transition probability of reaching this final state. This transition probability determines the absorption coefficient. In fact, a constructive wave interference pattern gives a higher transition probability (absorption coefficient) than destructive interference. This explains why the EXAFS structure, occurring as a modulation of the absorption coefficient, has its origin in the photoelectron wave interference pattern.

It is the interference pattern which presents the opportunity to determine the local structural parameters. Since interference is based on the interaction between the outgoing wave from an atom and the backscattered wave from its neighbour(s), the information about local structure is contained in the interference pattern and thus in the EXAFS structure. We will now describe how structural parameters can be extracted from this information and we will use the EXAFS spectrum shown in Fig. 2 as an example.

\section{Data analysis}

The analysis starts with subtraction of the background and normalization on a per atom basis 


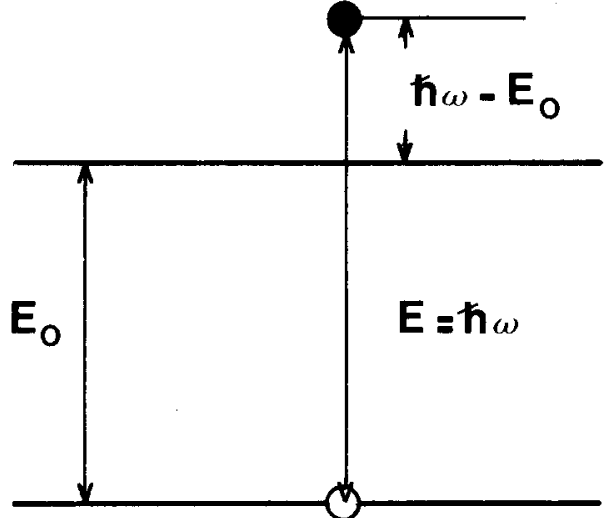

Fig. 3. Relation between the energy of the ejected electron and the photon energy, $\mathbf{E}=\hbar w$.

(via formula 1 ), resulting in the pure absorption fine structure $\chi(k)$ (Fig. 4a). This fine structure can now be approximated ${ }^{1}$ by a theoretical expression which is based upon the interference phenomenon extending for $j$ coordination shells:

$$
\chi(k)=\sum_{j} A_{j}(k) \sin \left[2 k R_{j}+2 \delta_{j}(k)\right],
$$

with

$$
A_{j}(k)=\frac{N_{j}}{k R_{j}^{2}} f_{j}(k) \exp \left(-2 R_{j} / \lambda\right) \exp \left(-2 k^{2} \sigma_{j}^{2}\right)
$$

a scattering factor, which is similar to the structure factor in X-ray diffraction. $R_{j}$ and $N_{j}$ are the distance to and the number of atoms in the $j$ 'th coordination shell; $\boldsymbol{\delta}_{j}(k)$ is a phase factor, which depends on both the absorbing and scattering atom; $f_{j}(k)$ is the amplitude factor determined by the type of scattering atom. The scattering factor $A_{j}(k)$ includes a term due to the elastic scattering of the photoelectron ( $\lambda$ is the mean free path for elastic scattering) and a pseudo Debye-Waller factor which includes both dynamic and static structural disorder.

The formulae 3-4 relate the EXAFS function $\chi(k)$ to the structural parameters $R_{j}, N_{j}$ and $\sigma_{j}$, which have to be extracted from the experimental EXAFS data. The next step is calculation of the radial distribution function $\phi(r)$, which gives the probability of finding a nucleus at a distance $r$ from the $\mathrm{X}$-ray absorbing atom. This radial distribution function is arrived at by Fourier transformation of the experimentally obtained fine structure $\chi(k)$ (via formula 1 ):

$$
\phi(r)=\frac{1}{\sqrt{ } 2 \pi} \int_{k_{\text {min }}}^{k_{\text {max }}} \chi(k) \exp (-2 i k r) \mathrm{d} k
$$

Fourier transformation of the fine structure $\chi(k)$ from Fig. 4 a results in the radial distribution function as shown in Fig. 4b. One should note that the maxima in the radial distribution function are shifted from the real interatomic distance $R_{j}$ by a factor $a_{j}$, which is determined by the phase function $\delta_{j}(k)$ in formula $(3)$ :

$$
r_{j}=R_{j}-a_{j}
$$

Owing to the elastic scattering of the photoelectron and to the factor $1 / R_{j}^{2}$ the fine structure $\chi(k)$ and thus the radial distribution function have a large contribution from the first coordination shell with decreasing contributions from other spheres.

Back Fourier transformation of only the first maximum in the radial distribution function gives the EXAFS function $\chi_{1}(k)$ originating solely from the first coordination shell. Information about $R_{1}, N_{1}$ and $\sigma_{1}$ can then be obtained when phase $\delta_{1}(k)$ and amplitude $f_{1}(k)$ are known. Theoretically calculated phase and amplitude factors have been used in the literature ${ }^{2}$. The factors $\delta(k)$ and $f(k)$ can also be derived from EXAFS measurements on calibration samples with the same absorbance and scatter combinations. The transfer of phase and amplitude has been shown to be feasible in most cases ${ }^{3}$. However, transferability of
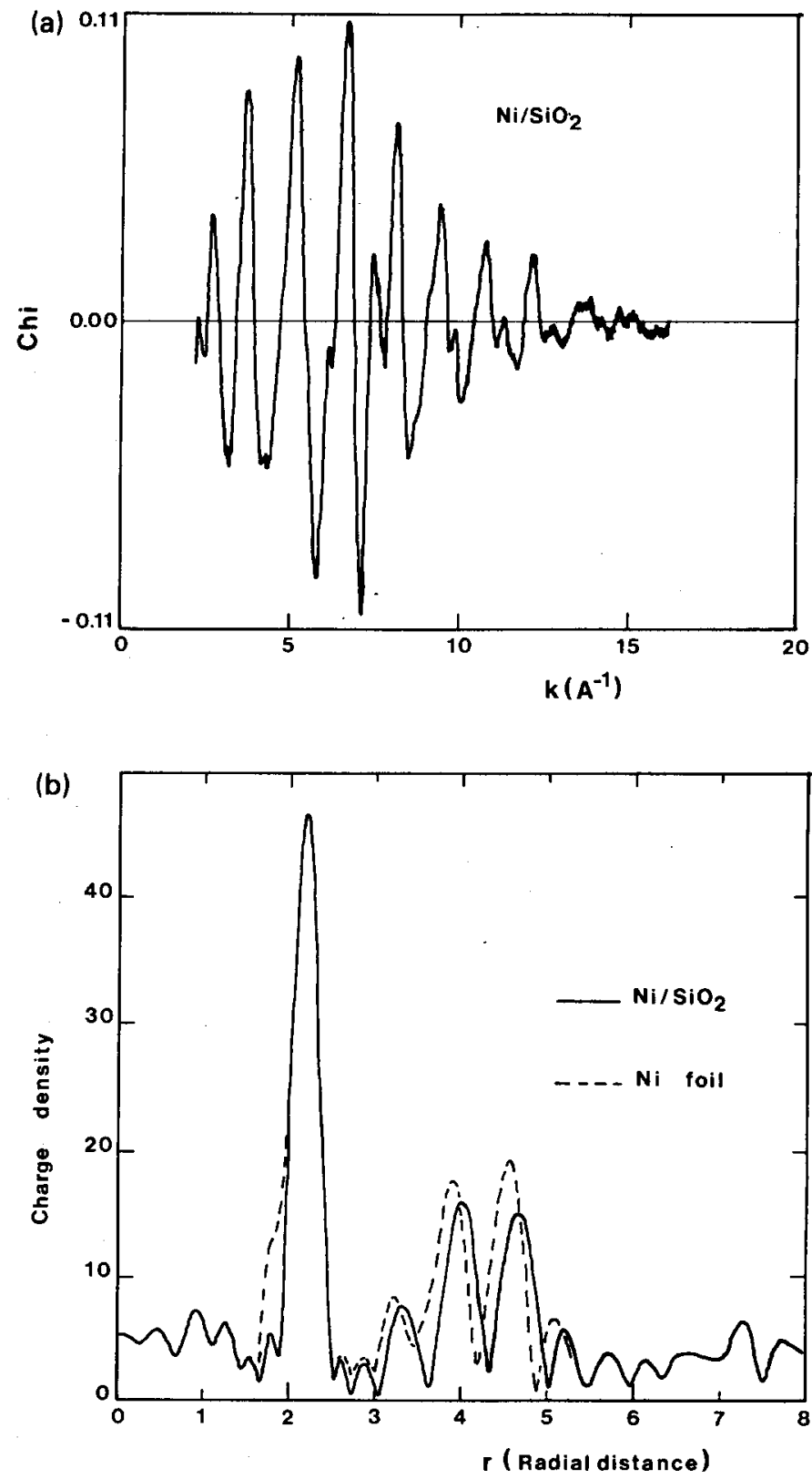

Fig. 4. Data analysis of the $X$-ray absorption spectrum of the nickel metal catalyst. (a) The fine structure $\chi(\mathbf{k})\left(\mathrm{Ni} / \mathrm{SiO}_{2}\right)$ afler background sublraction and normalization. (b) Radial distribution function obtained after Fourier transformation of $\chi(\mathbf{k})(\mathrm{Ni} / \mathrm{SiO})$. (The radial distribution function of $\mathrm{Ni}$ foil is given as a comparison.) 
TABLE I

\begin{tabular}{lccc}
\hline \multicolumn{1}{c}{ Method } & $\begin{array}{c}\text { Counts } \\
\left(\mathrm{s}^{-1}\right)\end{array}$ & $\begin{array}{c}\text { Resolution } \\
(\mathrm{eV})\end{array}$ & $\begin{array}{c}\text { Detection } \\
\text { limit } \\
\left(\mathrm{mol} \mathrm{l}^{-1}\right)\end{array}$ \\
\hline $\begin{array}{l}\text { Flat crystal } \\
+ \\
\text { X-ray } 1 \mathrm{~kW}\end{array}$ & $10^{3}$ & $15-20$ & Bulk \\
$\begin{array}{l}\text { Curved crystal } \\
+\end{array}$ & $10^{6}$ & $5-10$ & 2.5 \\
$\begin{array}{l}\mathrm{X} \text {-ray } 1 \mathrm{~kW} \\
\begin{array}{l}\text { Curved crystal } \\
+ \\
\text { Rotating anode }\end{array}\end{array}$ & $\geqslant 10^{7}$ & $5-10$ & $5 \times 10^{-1}$ \\
Storage ring & $10^{8}-10^{10}$ & 1 & $10^{-2}$ \\
\hline
\end{tabular}

amplitude factors might lead to serious mistakes in the number of nearest neighbours, when the chemical environment and/or valence state of the scattering atom are not the same in both cases.

In conclusion, it has to be mentioned that coordination distances for the first shell of neighbouring atoms can be determined with accuracies varying from 0.01 to $0.05 \AA$. The best obtainable accuracy in the determination of the number of nearest neighbour atoms is $10-20 \%$. The procedure for EXAFS data analysis described above can only be used to obtain reliable information for the first coordination shell. This limitation is mainly a result of the assumptions which are made in the derivation of formula (3). In the future improvements in the method may be implemented and in this connection the approach taken by Lee \& Pendry ${ }^{4}$ which can lead to a useful analysis up to five coordination shells, is worth mentioning.

\section{Synchrotron radiation or in-house facilities}

By using a conventional low power X-ray tube, a crystal Bragg monochromator and a film as detector, as early as the 1930s $\mathrm{Kronig}^{5}$ observed (and partly explained) the EXAFS oscillations. In the late 1960s new detector and digitalization techniques led to measurements with a better signal-to-noise ratio. However, scan times of sometimes more than one week were not unusual.

Extremely important for the development of the EXAFS technique was the fact that Sayers, Stern and Lytle $^{1}$ showed, by using a particle-in-box model, that EXAFS could be used as a tool for determination of local structure. Since then interest in EXAFS has increased tremendously due to further development of the theory ${ }^{6}$ and due to the fact that in the seventies synchrotron radiation, produced by high energy storage rings, became available. The high intensity of synchrotron radiation enables EXAFS experiments to be done with much better signal-to-noise ratios which are obtained in a much shorter time than with the use of conventional X-ray tubes.

Scientists working in countries where synchrotron radiation facilities are not available, or scientists who have limited access to these facilities (usually due to the overcharging for beamtime) still have the opportunity to build their own in-house instrument with an X-ray tube as light source. Count rates obtained with different kinds of configurations are given in Table I. Synchrotron radiation is essential in those cases where very dilute materials have to be studied, but specially designed in-house instruments are capable of handling many problems.

By using a rotating anode Röntgen generator, a curved monochromator crystal of the Johannson type and a Rowland circle configuration, EXAFS spectra can be measured within a reasonable amount of time ${ }^{7}$ (15-30 $\mathrm{min})$. Since the X-ray generator must remain fixed, the wavelength of the photon beam reflected by the curved monochromator crystal has to be selected by changing the distance between the crystal and the $\mathrm{X}$-ray source. The energy resolution which can be obtained with such a set-up depends on the quality of the curved monochromator crystal and the resetability of the movable parts of the spectrometer. The best value has been estimated in the literature to be $10-20 \mathrm{eV}^{8}$. This is sufficient for detecting the fine structure $\chi(k)$ with a reasonable resolution.

\section{Applications}

For solid materials with a high degree of short-range order but lacking long range order, EXAFS is the best method available for structural determination. In disordered solids (as in $\mathrm{Si}$ ) amorphous solids (such as films and glasses) or semi-conductors (such as

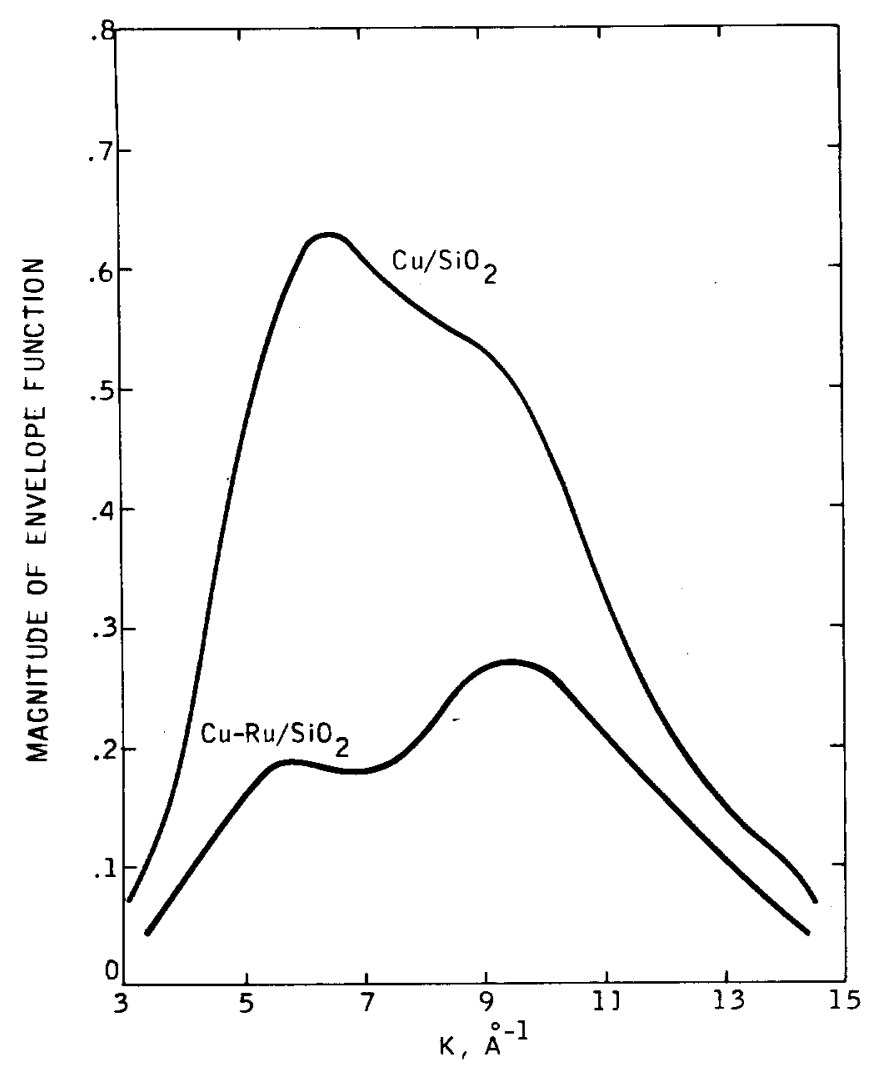

Fig. 5. Influence of ruthenium on the envelope function of the $\mathrm{Cu}$ fine structure $\chi(\mathbf{k})$ measured on a copper ruthenium catalyst ${ }^{10}$. 
$\left.\mathrm{Cu}_{x}\left(\mathrm{As}_{4} \mathrm{Se}\right)_{1-x}\right)$ and metals (PdGe), EXAFS can uniquely determine nearest neighbour distances, coordination numbers and mean-square relative displacements ${ }^{9}$. EXAFS can prove very helpful, even in the study of crystalline materials, since in usual diffraction methods the arrangements of atoms in the unit cell must be obtained from intensity measurements, which are rather difficult to analyse.

As an example of the application of EXAFS to disordered solids we present here some results of EXAFS measurements of metal-on-support catalysts. The average metal particle size $(\mathrm{d}<40 \AA)$ of highly dispersed metal catalysts makes the application of normal diffraction techniques to structural studies impossible. For the ruthenium copper system chemisorption and catalytic studies led to the idea of the presence of bimetallic ruthenium-copper clusters. Essential detailed structural information was obtained with EXAFS $^{10}$.

Fig. 5 gives the envelope function (in fact $A_{1}(k)$, formula 3) of the first coordination shell of $\mathrm{Cu}$ in the

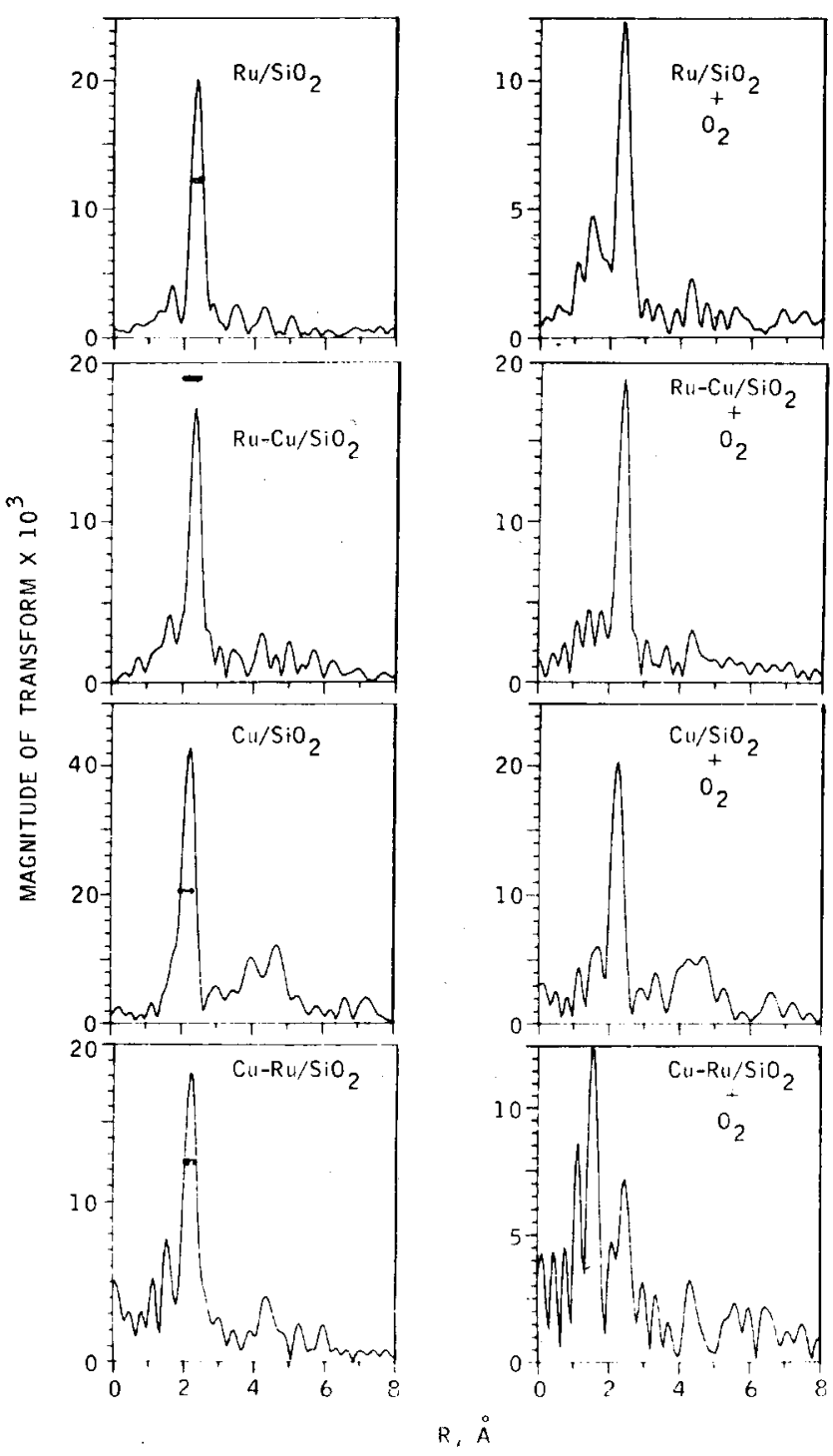

Fig. 6. Influence of oxygen on the radial distribution functions of the copper nuthenium catalyst and the $\mathrm{Cu} / \mathrm{SiO}_{2}$ and $\mathrm{Ru} / \mathrm{SiO}_{2}$ reference catalysts ${ }^{10}$.
$\mathrm{Cu} / \mathrm{SiO}_{2}$ and $\mathrm{CuRu} / \mathrm{SiO}_{2}$ catalysts $(\mathrm{Cu} / \mathrm{Ru}=1 / 1)$, respectively. $\mathrm{Ru}$ has a marked influence on the coordination of $\mathrm{Cu}$ in the bimetallic catalyst. The influence of $\mathrm{Cu}$ on the nearest neighbour shell of $\mathrm{Ru}$ in the bimetallic catalysts is much less pronounced. Chemisorption of oxygen at room temperature (1\% oxygen in helium) provided further information. The right-hand side of Fig. 6 shows the effect of oxygen on the radial distribution function of the catalyst and its reference compounds $(\mathrm{Ru}-\mathrm{Cu}$ and $\mathrm{Cu}-\mathrm{Ru}$ indicate the $\mathrm{Ru}$-edge and the $\mathrm{Cu}$-edge in the bimetallic catalyst, respectively). Oxygen influences the radial distribution functions of the $\mathrm{Cu}-\mathrm{Ru} / \mathrm{SiO}_{2}, \mathrm{Cu} / \mathrm{SiO}_{2}$ and $\mathrm{Ru} / \mathrm{SiO}_{2}$ catalysts drastically. The intensity of the peak from the first coordination shell of $\mathrm{Ru}$ in the $\mathrm{Ru}-\mathrm{Cu} / \mathrm{SiO}_{2}$ catalysts does not change (within the uncertainty limits) upon oxygen chemisorption. Further analysis resulted in a structural model of bimetallic clusters with a core of ruthenium atoms covered by copper. Best fit values for the compositions of the first coordination shells about copper and ruthenium were found to be 52 and $92 \%$ ruthenium.

Another field of application for EXAFS is that of local structures. Thus the environment of ions in solution, of impurities in solids and at surfaces, and of atoms in molecules can be determined. In this way ionpairing in aqueous solutions at high concentrations has been measured ${ }^{11}$ for $\mathrm{Cu}^{2+}$ and $\mathrm{Br}^{-}$and also the hydrolytic polymerization of $\mathrm{Fe}^{3}+$ in aqueous solution $^{12}$ in $\left(\mathrm{H}_{2} \mathrm{O}\right)_{4} \mathrm{Fe}(\mathrm{OH})_{2} \mathrm{Fe}\left(\mathrm{H}_{2} \mathrm{O}\right)_{4}^{4+}$. A example of the determination of the local structure of an atom in a molecule is that of the homogeneous catalyst $\left(\mathrm{Ph}_{3} \mathrm{P}\right)_{3} \mathrm{RhCl}$, chlorotris(triphenylphosphine)rhodium. This industrially important catalyst is used in the hydrogenation of many organic molecules. To improve its separation from reactants and products it had been tried to heterogenize this catalyst with polystyrene-divinylbenzene as a polymeric support. This resulted, however, in a significant reduction in catalytic activity. A structural study with X-ray diffraction failed to give any information due to the lack of crystallinity of the supported material and the scattering of the support itsclf. The results of the EXAFS measurements ${ }^{13}$ on the supported catalyst are given in Fig. 7b. The EXAFS structural data for the monomeric free catalyst (Fig. 7a) correspond to those obtained with X-ray diffraction of single crystals. The decrease in the number of $\mathrm{Rh}-\mathrm{P}_{2}$ bonds $(2 \rightarrow 1)$ and the increase of the number of Rh-Cl bonds $(1 \rightarrow 2)$ indicate dimerization of the monomeric $\mathrm{Rh}$ complex, which also explains the decrease in catalytic activity.

Many examples of the EXAFS determination of the environment of atoms in (large) molecules (biochemical materials and especially metal-enzymes) have already been given in literature. Two outstanding examples will be mentioned here. EXAFS measurements of oxyhemoglobin, deoxyhemoglobin and of related model compounds ${ }^{14}$ have shown that the bond length between the iron atom and the porphyrin nitrogen atoms does not change on oxygenating 


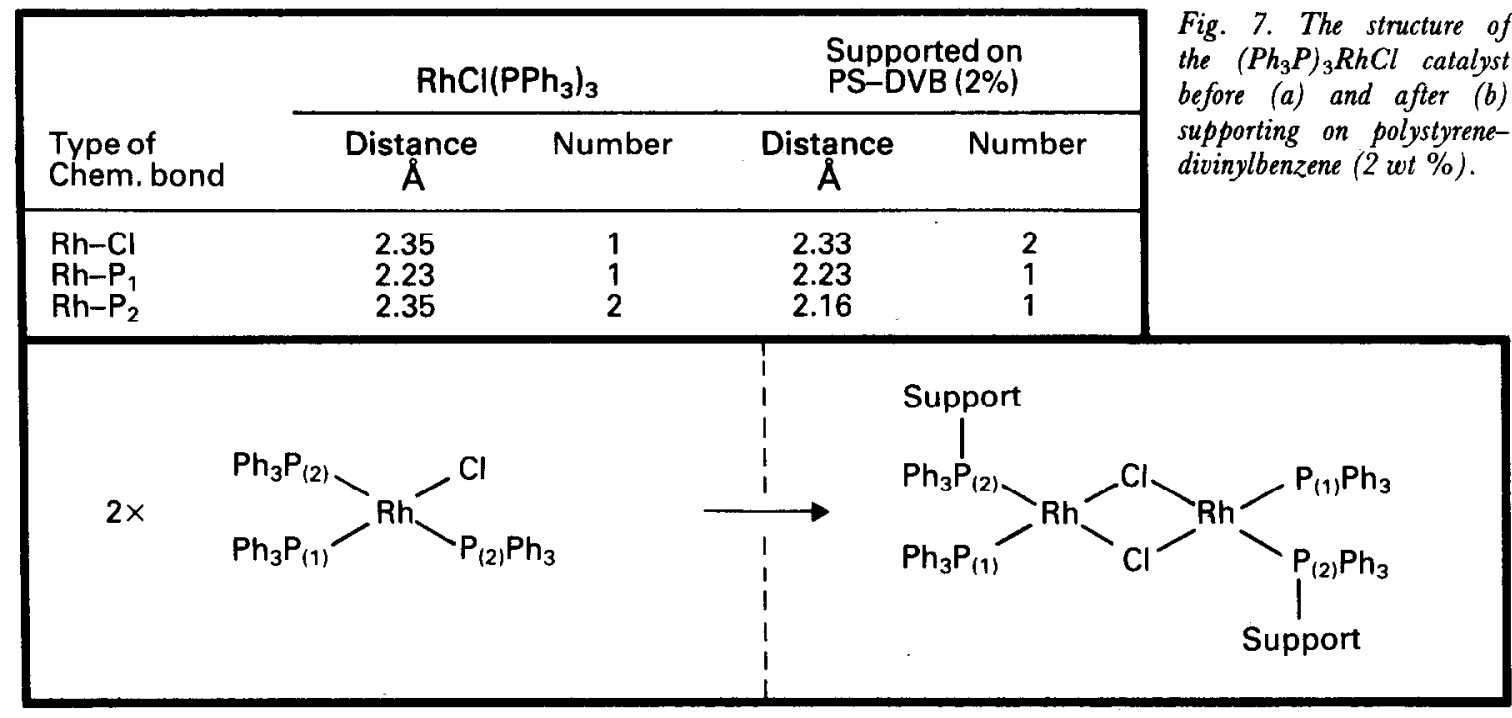

(a)

(b)

hemoglobin. This means that there can be no strain energy around the iron in the enzyme. Cooperative changes in the total structure of hemoglobin on oxygenation and deoxygenation cannot be caused by differences in the iron environment. This is in disagreement with a model proposed by Hoard and Perutz and calls for another look at the problem of the function and structure of hemoglobin.

Another biochemical problem in which EXAFS played a vital role is that of the molybdenum-iron protein of the nitrogenase enzyme. Despite the fact that many techniques had been tried before, only EXAFS, using synchrotron radiation has offered the possibility of determining the position of molybdenum in the protein. This work on nitrogenase has stimulated an upsurge of synthetic inorganic research on analogs of the molybdenum site and the work on iron, molybdenum, sulfur cubanes by Holm et al. ${ }^{15}$ has to be mentioned in this respect.

To conclude, in the last few years EXAFS has had an enormous impact on determination of local structure and short range order. The applications mentioned above are just a few of the many examples already published. Many more applications of the EXAFS technique are to be expected in the near future. As a result of this interest, more knowledge on the building of in-house facilities will be gained and it can be foreseen that a sizeable number of such instruments will become available.

\section{References}

1 Sayers, D. E., Lytle, F. W. and Stern, E. A. (1970) Adv, X-ray Anal. 13, 248; Stern, E. A. (1974) Phys. Rev. B, 10, 3027; Lytle, F. W., Sayers, D. E. and Stern, E. A. (1975) Phys. Rev. B, 11, 4825; Stern, E. A., Sayers, D. E. and Lytle, F. W. ibid., p. 4836

2 Lee, P. A. and Beni, G. (1977) Phys. Rev. B, 15, 2862; Teo, B. K. and Lee, P. A. (1979) J. Am. Chem. Soc. 101, 2815

3 Cramer, S. P., Eccles, T. K., Kutzler, F., Hodgson, K. O. and Doniach, S. (1976) J. Am. Chem. Soc. 98, 8059; Teo, B. K., Lee, P. A., Simons, A. L., Eisenberger, P. and Kincaid, B. M. (1977) J. Am. Chem. Soc. 99, 3854; Lee, P. A., Teo, B. K. and Simons, A. L. (1977) J. Am. Chem. Soc. 99, 3859
4 Lee, P. A. and Pendry, J. B. (1975) Phys. Rev. B 11, 2795

5 Kronig, R. L. (1931) Z. Phys. 70, 317; ibid. (1932) 75, 191; ibid. (1932) 75,468

6 Ashley, C. A. and Doniach, S. (1975) Phys. Rev. B, 11, 1279; Stern, E. A., Bunker, B. A. and Heald, S. M. (1980) Phys. Rev. $\mathrm{B}, 21,5521$

7 Del Cueto, J. A. and Shevchik, N. J. (1978) J. Phys. E, 11, 616; Robinson, A. L. (1979) Science 205, 1367

8 Knapp, G. S., Chen, H. and Klippert, T. E. (1978) Rev. Sci. Instrum. 49, 1658; Georgopoulos, P. and Knapp, G. S. submitted to J. Appl. Cryst.

9 Brown, G. S. (1980) in H. Winick and S. Doniach, Synchrotron Radiation Research, Plenum Press, New York, Ch. 11, p. 387

10 Sinfelt, J. H., Via, G. H. and Lytle, F. W. (1980) J. Chem. Phys. 72,4832

11 Eisenberger, P. and Kincaid, B. M. (1977) Chem. Phys. Lett. 36, 134

12 Morrison, T. I., Reis, A. H., Knapp, G. S., Fradin, F. Y., Chen, H. and Klippert, T. E. (1978) J. Am. Chem. Soc. 100, 3262

13 Reed, J., Eisenberger, P., Teo, B. K. and Kincaid, B. M. (1977) J. Am. Chem. Soc. 99, 5218; Reed, J. and Eisenberger, P., Teo, B. K. and Kincaid, B. M. (1978) J. Am. Chem. Soc. 100, 2375

14 Doniach, S., Eisenberger, P. and Hodgson, K. O. (1980) in H. Winick and S. Doniach, Synchrotron Research, Plenum Press, New York, Ch. 13, p. 425

15 Wolff, T. E., Berg, J. M., Warrick, C., Holm, R. H., Hodgson, K. O. and Fraenkel, R. B. (1978) J. Am. Chem. Soc. 100, 4030

\section{Acknowledgement}

One of us (DCK) wishes to express his gratitude for the hospitality received during his stay (spring 1979) in S.S.R.L. (Stanford University) and in C.C.S.T. (University of Delaware), U.S.A. The discussions with Prof. Katzer and Prof. Sayers are gratefully acknowledged.

R. Prins receized his Ph.D. in physical chemistry in 1967. He worked at the Shell Laboratories in Amsterdam, The Netherlands, for eleven years, first in the fundamental catalysis department of Prof. Dr W. H. M. Sachtler and later in applied research on metal catalysts. He is now professor of Inorganic Chemistry and Catalysis, Eindhoven, University of Technology, The Netherlands.

With a Ph.D. degree in Physics D. C. Koningsberger joined the research group of Prof. Dr G. C. A. Schuit (University of Technology, Eindhoven, The Netherlands) in 1972. He was mainly concerned with structure determination of catalyticmaterials. He gained experience with the EXAFS technique during his stay (spring 1979) in S.S.R.I. (Stanford Vniversity) and in C.C.S.T. (University of Delaware), U.S.A. 